\title{
The Gribov mode in hot QCD
}

\author{
Nan Su${ }^{1, a}$ \\ ${ }^{1}$ Frankfurt Institute for Advanced Studies, 60438 Frankfurt am Main, Germany
}

\begin{abstract}
In this proceedings, I summarize recent findings of a novel massless mode, dubbed as "Gribov mode", generated by the (chromo)magnetic scale $g^{2} T$ in hot QCD. The Gribov mode is a genuine non-Abelian mode inducing effects such as positivity violation.
\end{abstract}

\section{Introduction}

Gauge fixing is a mathematical procedure for removing redundant degrees of freedom in the field variables of gauge theories. A popular gauge-fixing procedure was invented by Faddeev and Popov [1] that leads to satisfactory results for Abelian gauge theories. However in the seminal work of Ref. [2], Gribov pointed out that there are still residue redundant degrees of freedom - namely the Gribov copies - after the Faddeev-Popov procedure. The Gribov copies reside in the IR of the non-Abelian gauge fields, and they are intimately related to the confinement of color charges. Later on, Zwanziger generalized Gribov's semi-classical approach to all orders that gave birth to the Gribov-Zwanziger action [3]. The Gribov-Zwanziger scenario has stimulated flourishing developments in the study of color confinement (see Refs. [4, 5] for reviews).

Since the last decade, there has been an increasing effort in generalizing the Gribov-Zwanziger scenario to finite temperature stimulated by the Linde problem that invalids conventional thermal perturbation theory at the (chromo)magnetic scale $g^{2} T[6,7]$. The non-perturbative nature of the magnetic scale is intimately relathard-thermal-looped to the confining property of the dimensionally reduced Yang-Mills theory at high temperature. This suggests the need of incorporating a confinement mechanism in perturbative expansions even when dealing with the deconfined quark-gluon plasma phase. The Gribov-Zwanziger action provides an ideal framework for this purpose. It regulates the IR behavior of QCD by fixing the Gribov copies that remain after applying the Faddeev-Popov procedure. The Gribov-Zwanziger action is renormalizable, therefore it provides a systematic framework for perturbative calculations (i.e., $g \ll 1$ ) incorporating confinement effects. The gluon propagator in general covariant gauge reads

$$
D^{\mu \nu}(P)=\left[\delta^{\mu \nu}-(1-\xi) \frac{P^{\mu} P^{\nu}}{P^{2}}\right] \frac{P^{2}}{P^{4}+\gamma_{G}^{4}},
$$

where $\xi$ is the gauge parameter. The Gribov parameter $\gamma_{G}$ is solved self-consistently from a gap equation that is defined to infinite loop orders. The Gribov-Zwanziger gluon propagator is IR suppressed,

\footnotetext{
a e-mail: nansu@ fias.uni-frankfurt.de
} 
manifesting confinement effects, and it is a significant improvement over the one from the FaddeevPopov quantization which forms the basis of conventional perturbative calculations. The gap equation at one-loop order can be solved analytically at asymptotically high temperatures and gives [8]

$$
\gamma_{G}=\frac{D-1}{D} \frac{N_{c}}{4 \sqrt{2} \pi} g^{2} T,
$$

where $D$ is the space-time dimensions and $N_{c}$ is the number of colors. Eq. (2) provides a fundamental IR cutoff at the magnetic scale for the finite-temperature Gribov-Zwanziger action. In this way, the magnetic scale is intrinsically embedded in the Gribov-Zwanziger action.

\section{Results and discussions}

Self-energies of quarks and gluons are important measures for the collective behavior of the quarkgluon plasma, since thermal masses, dispersion relations, and spectral functions of collective excitations are derived from them. The Euclidean one-loop quark self-energy reads

$$
\Sigma(P)=(i g)^{2} C_{F} \oint_{\{K\}} \gamma^{\mu} S(K) \gamma^{v} D^{\mu v}(P-K),
$$

where $S(P)$ is the quark propagator and $D^{\mu v}(P)$ is the gluon propagator taken from Eq. (1). It is worth noting that there have been similar studies for the quark self-energy with non-perturbative gluons at finite density $[9,10]$ and in strong magnetic fields [11].

At $g \ll 1$ (i.e., high temperatures), we may apply the hard-thermal-loop systematics [13] in analyzing Eq. (3). As a result, the gauge-invariant contribution to Eq. (3) reads [12]

$$
\begin{aligned}
\Sigma(P) \simeq & -(i g)^{2} C_{F} \sum_{ \pm} \int_{0}^{\infty} \frac{\mathrm{d} k}{2 \pi^{2}} k^{2} \int \frac{\mathrm{d} \Omega}{4 \pi} \frac{\tilde{n}_{ \pm}\left(k, \gamma_{G}\right)}{4 E_{ \pm}^{0}} \\
& \times\left[\frac{i \gamma_{0}+\hat{\boldsymbol{k}} \cdot \boldsymbol{\gamma}}{i P_{0}+k-E_{ \pm}^{0}+\frac{\boldsymbol{p} \cdot \boldsymbol{k}}{E_{ \pm}^{0}}}+\frac{i \gamma_{0}-\hat{\boldsymbol{k}} \cdot \boldsymbol{\gamma}}{i P_{0}-k+E_{ \pm}^{0}-\frac{\boldsymbol{p} \cdot \boldsymbol{k}}{E_{ \pm}^{0}}}\right],
\end{aligned}
$$

where $\hat{\boldsymbol{k}}=\boldsymbol{k} / k$ with $k=|\boldsymbol{k}|, E_{ \pm}^{0}=\sqrt{k^{2} \pm i \gamma_{G}^{2}}, \tilde{n}_{ \pm}\left(k, \gamma_{G}\right) \equiv n_{B}\left(\sqrt{k^{2} \pm i \gamma_{G}^{2}}\right)+n_{F}(k)$ with $n_{B}$ and $n_{F}$ the Bose-Einstein and Fermi-Dirac distributions, and $\int \mathrm{d} \Omega=\int_{0}^{2 \pi} \mathrm{d} \phi \int_{0}^{\pi} \mathrm{d} \cos \theta$.

The quark thermal mass incorporating effects from $g^{2} T$ reads

$$
m_{q}^{2}\left(\gamma_{G}\right)=\frac{g^{2} C_{F}}{4 \pi^{2}} \sum_{ \pm} \int_{0}^{\infty} d k \frac{k^{2} \tilde{n}_{ \pm}\left(k, \gamma_{G}\right)}{E_{ \pm}^{0}}
$$

It reduces to the conventional hard-thermal-loop one, $m_{q}^{2}(0)=C_{F} g^{2} T^{2} / 8$, when setting $\gamma_{G}=0 . m_{q}^{2}\left(\gamma_{G}\right)$ receives negative contributions from $\gamma_{G}$, which is a manifestation of anti-screening effects generated by $g^{2} T$ (see Fig. 1 in Ref. [12] for details). This is a profound signal of the build-up of long-range correlations in the system.

The dispersion relation is obtained by analytically continuing the self-energy (4) to Minkowski space and then solving the poles in the corresponding quark propagator $i S^{-1}(P)=\not P-\Sigma(P)=0$. In contrast to the conventional hard-thermal-loop case, there are three poles in the propagator (see Fig. 2 in Ref. [12] for details). Firstly, the screened quasi-particle excitations are recovered,

$$
\omega=\omega_{+}\left(p ; \gamma_{G}\right), \quad \omega=\omega_{-}\left(p ; \gamma_{G}\right),
$$


which are the so-called particle $\omega_{+}$and plasmino $\omega_{-}$modes, with $\omega_{ \pm}\left(0 ; \gamma_{G}\right)=m_{q}\left(\gamma_{G}\right)$ as expected. Both $\omega_{ \pm} / m_{q}\left(\gamma_{G}\right)$ and their residues $Z_{ \pm}$are $g$-independent in the studied range. This property is identical to the conventional hard-thermal-loop case, which provides a non-trivial consistency check of the setup. Furthermore, there exists a novel excitation named Gribov pole as in Ref. [12],

$$
\omega=\omega_{G}\left(p ; \gamma_{G}\right) \text {. }
$$

It describes massless fermionic excitations in the quark-gluon plasma with dispersion relation $\omega=v_{s} p$ at small momenta, with $v_{s} \approx 1 / \sqrt{3}$ (speed of sound) independent of $g$ for the studied range. The Gribov mode "grows" in the $(\omega, p)$-plane while increasing the magnetic scale, and this effectively introduces a new magnetic scaling behavior to the non-Abelian plasma. At larger momenta than the permitted ones for each coupling, we hit branch cuts and Landau damping consequently takes place. The Gribov pole goes along with a residue $Z_{G}(p)<0$ that induces positivity violation in the corresponding spectral functions in the region of space-like momenta. These novel features are direct manifestations of long-range confinement effects surviving at finite $T$ in the non-Abelian plasma. The results reflect common features of Gribov-like approaches [2, 3, 14], though the calculation was done via the Gribov-Zwanziger action. It is tempting to explore the impact of the setup to heavy-ion phenomenology [15-18]. It is also interesting to understand whether there are any relations between the Gribov mode and the QCD transition.

\section{Acknowledgments}

This proceedings is based on the work in collaboration with Konrad Tywoniuk. I acknowledge the support from the GSI Helmholtzzentrum für Schwerionenforschung and Horst Stöcker.

\section{References}

[1] L. D. Faddeev and V. N. Popov, Phys. Lett. B 25, 29 (1967).

[2] V. N. Gribov, Nucl. Phys. B 139, 1 (1978).

[3] D. Zwanziger, Nucl. Phys. B 323, 513 (1989).

[4] Y. L. Dokshitzer and D. E. Kharzeev, Ann. Rev. Nucl. Part. Sci. 54, 487 (2004).

[5] N. Vandersickel and D. Zwanziger, Phys. Rept. 520 (2012) 175.

[6] A. D. Linde, Phys. Lett. B 96, 289 (1980).

[7] D. J. Gross, R. D. Pisarski and L. G. Yaffe, Rev. Mod. Phys. 53, 43 (1981).

[8] K. Fukushima and N. Su, Phys. Rev. D 88, 076008 (2013).

[9] T. Kojo, Y. Hidaka, L. McLerran and R. D. Pisarski, Nucl. Phys. A 843, 37 (2010).

[10] T. Kojo, Y. Hidaka, K. Fukushima, L. D. McLerran and R. D. Pisarski, Nucl. Phys. A 875, 94 (2012).

[11] T. Kojo and N. Su, Phys. Lett. B 720, 192 (2013).

[12] N. Su and K. Tywoniuk, Phys. Rev. Lett. 114, 161601 (2015).

[13] E. Braaten and R. D. Pisarski, Nucl. Phys. B 337, 569 (1990).

[14] D. Dudal, J. A. Gracey, S. P. Sorella, N. Vandersickel and H. Verschelde, Phys. Rev. D 78, 065047 (2008).

[15] W. Florkowski, R. Ryblewski, N. Su and K. Tywoniuk, Acta Phys. Polon. B 47, 1833 (2016).

[16] A. Bandyopadhyay, N. Haque, M. G. Mustafa and M. Strickland, Phys. Rev. D 93, 065004 (2016).

[17] W. Florkowski, R. Ryblewski, N. Su and K. Tywoniuk, Phys. Rev. C 94, 044904 (2016).

[18] V. Begun, W. Florkowski and R. Ryblewski, arXiv:1602.08308 [nucl-th]. 